\title{
Pulsed Eddy Current Non-destructive Testing and Evaluation: A Review
}

\author{
Ali Sophian' ${ }^{1}$ Guiyun $\operatorname{Tian}^{2,3} \cdot{\text { Mengbao } \text { Fan }^{4}}^{4}$
}

Received: 2 November 2016/Revised: 18 January 2017/Accepted: 28 March 2017/Published online: 17 April 2017

(C) Chinese Mechanical Engineering Society and Springer-Verlag Berlin Heidelberg 2017

\begin{abstract}
Pulsed eddy current (PEC) non-destructive testing and evaluation (NDT\&E) has been around for some time and it is still attracting extensive attention from researchers around the globe, which can be witnessed through the reports reviewed in this paper. Thanks to its richness of spectral components, various applications of this technique have been proposed and reported in the literature covering both structural integrity inspection and material characterization in various industrial sectors. To support its development and for better understanding of the phenomena around the transient induced eddy currents, attempts for its modelling both analytically and numerically have been made by researchers around the world. This review is an attempt to capture the state-of-the-art development and applications of PEC, especially in the last 15 years and it is not intended to be exhaustive. Future challenges and opportunities for PEC NDT\&E are also presented.
\end{abstract}

Keywords Non-destructive testing · Pulsed eddy currents · Material characterization - Structural integrity ·

Non-destructive evaluation

Guiyun Tian

g.y.tian@uestc.edu.cn

1 Faculty of Engineering, International Islamic University Malaysia, Kuala Lumpur, Malaysia

2 School of Automation Engineering, University of Electronic Science and Technology of China, Chengdu, China

3 School of Electrical and Electronic Engineering, Newcastle University, Newcastle upon Tyne NE1 7RU, UK

4 School of Mechatronic Engineering, China University of Mining and Technology, Xuzhou 221116, Jiangsu, China

\section{Introduction}

Despite its approximately-five-decade-long history, PEC is still considered by many as a new emerging eddy current NDT\&E technique. Compared to other eddy current testing (ECT) techniques this view can be true. Literature shows that PEC has been attracting the attention of researchers from around the globe, including countries, such as China, UK, Canada, Portugal, USA, South Korea, Japan, France, Slovakia, Poland, and Italy.

The amount of attention that PEC NDT\&E has been receiving owes to the key potential benefits that it offers. The first and main advantage is that, compared to single frequency ECT, PEC inherently has a broadband of frequencies [1], which is advantageous for any eddy-currentbased NDT\&E techniques due to the frequency-dependant skin effect. Another benefit is that PEC signals are relatively easier to interpret, while it requires a special skill of the operators for interpreting conventional ECT signals which are presented in the impedance plane trajectory.

Conventional ECT only applies a single frequency for excitation which makes it unable to detect both surface and sub-surface defects reliably. The improved technique is the multi-frequency ECT which applies different excitation frequencies, one after another. Compared to multi-frequency ECT, PEC can potentially be applied in shorter time for inspection of different depths as PEC applies a wideband of frequencies in a single pulse. This allows to reduce the measurement time to the minimum one depending on the sample characteristics. Fig. 1 provides the illustration of the excitation waveforms of each of the methods.

Similar to other ECT techniques, in general PEC requires no surface preparation which leads to reduction of inspection time and costs efficiency is improved. The 

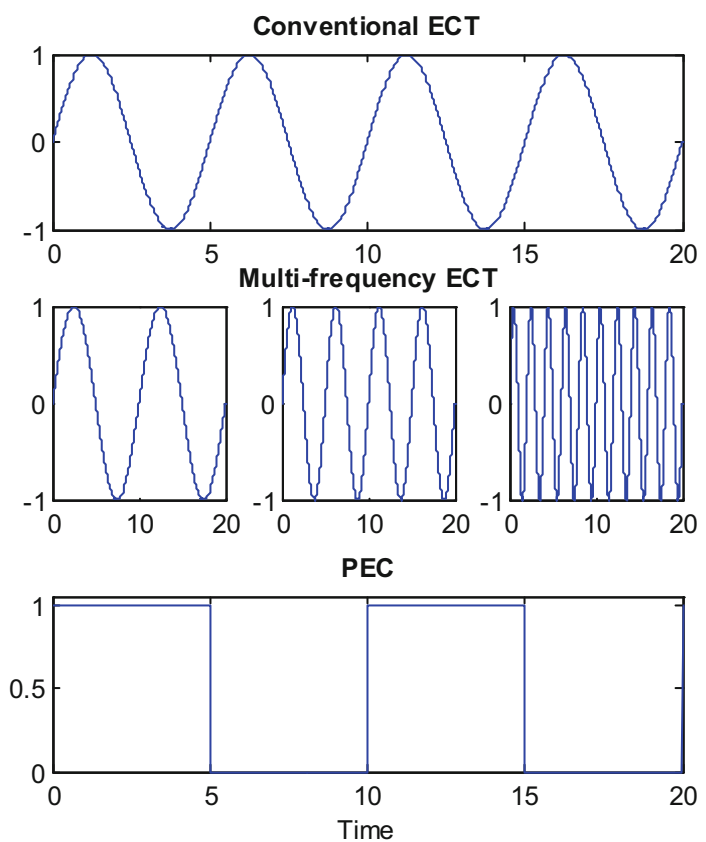

Fig. 1 Illustration of excitation waveforms for different ECT techniques

inspection can also be done without interrupting the operation or service of the structure being tested, unlike for example X-ray testing. In many applications where the sample is coated, no removal of the coating is required when ECT NDT\&E is used. Any eddy-current systems are relatively cost-effective and reliable.

In the following sections, the concept of PEC is briefly discussed which is then followed by the review in systems, modelling, signal processing and applications. A conclusion completes this review paper.

\section{Concept of Pulsed Eddy Current}

In eddy current NDT, an AC-driven excitation coil induces eddy current in the sample through electromagnetic coupling. In turn, the circulation of the eddy current induces a secondary magnetic field as illustrated Fig. 2. This field will vary if flaw that impedes the eddy currents is present or there is a change in the electrical conductivity, magnetic permeability or thickness of the sample. The change in the field will be picked up by a sensing device, which is typically either a coil or a magnetic sensor.

The penetration and the density of the eddy current in the sample is an important issue in any ECT. The penetration is limited due to the skin effect, which causes its density to decrease exponentially with depth. The depth at which the density has reduced to $1 / e$ of the density at the surface is termed the skin depth $\delta$ and defined by

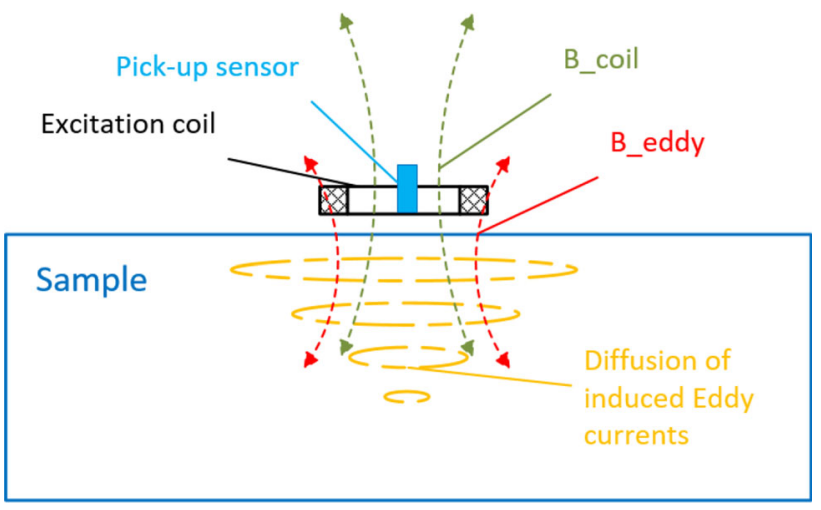

Fig. 2 Illustration of the working principle of ECT

$\delta=\sqrt{\frac{2}{\varpi \mu \sigma}}$,

where $\delta$ is skin depth (m), $\mu$ is magnetic permeability $(\mathrm{H} /$ $\mathrm{m}), \sigma$ is electrical conductivity $(\mathrm{S} / \mathrm{m})$ and $\omega$ is angular frequency $(\mathrm{rad} / \mathrm{s})$. The equation shows that the depth of penetration depends on the excitation frequency. The lower the frequency, the deeper the penetration and vice versa. In contrast to conventional sinusoidal eddy current technique, where the excitation is limited to one frequency component, pulsed eddy current techniques excite the induction coil with a pulse waveform. The frequency components of pulse waveform can be demonstrated using Fourier Transform. If the excitation waveform is defined as

$f(t)= \begin{cases}A, & -\frac{T}{2} \leq t \leq \frac{T}{2}, \\ 0, & |t|>\frac{T}{2},\end{cases}$

where $A$ is the amplitude of the pulse and $T$ is the pulse width, then using the amplitude spectrum of the excitation is defined as

$F(\omega)=\frac{2 \sin \omega T / 2}{\omega}$.

Fig. 3 shows examples of the pulses with two different widths and their power spectra, which shows that the excitation has a series of frequency components, which has given the technique the potential to inspect different depths simultaneously and therefore it will be able to offer more information compared to the conventional approach.

\section{PEC Systems}

Despite variations that exist, a typical PEC system will look like the illustration shown in Fig. 4. A pulse signal at a chosen frequency and pulse width is generated which is then power-amplified to drive an excitation coil. In turn, a 

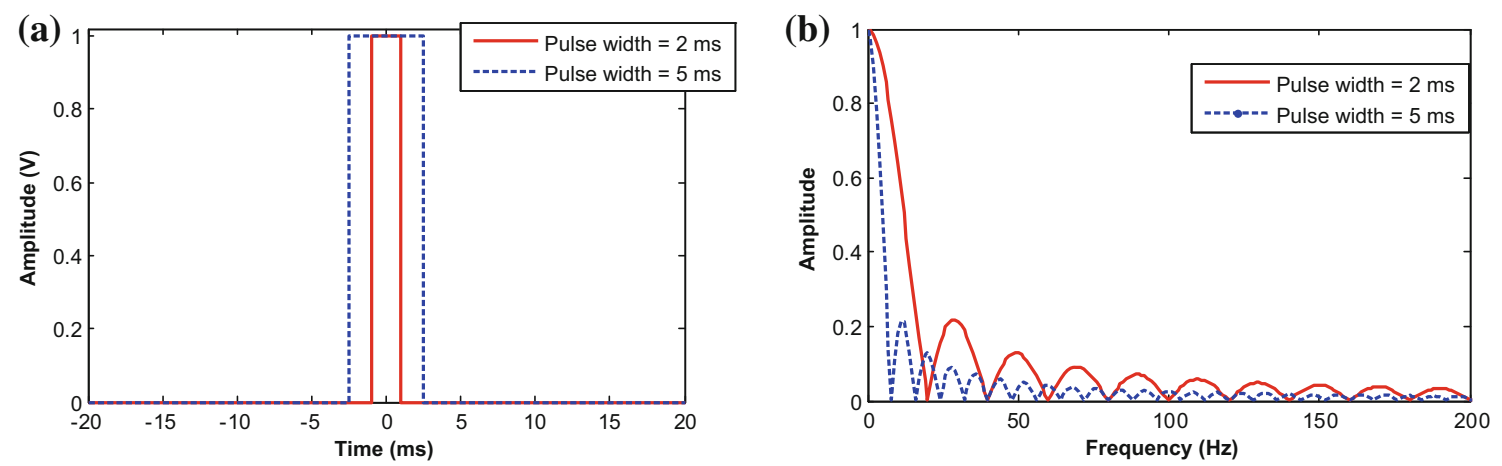

Fig. 3 (a) Examples of pulses with different widths, (b) Power spectra of the pulses

Fig. 4 Generic configuration of a PEC NDT system

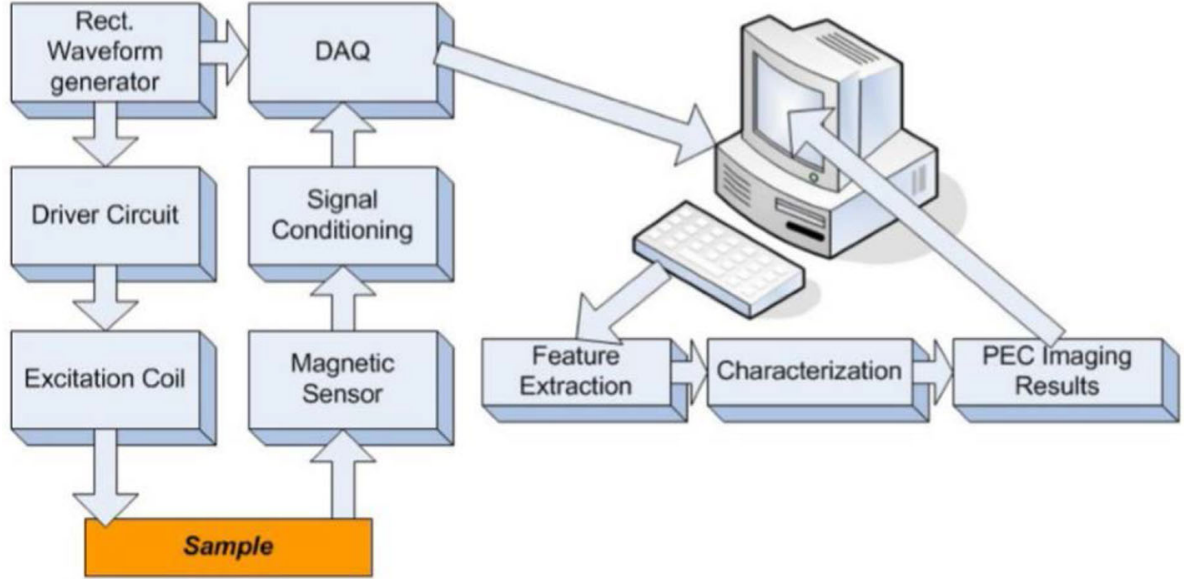

time-varying magnetic field is induced by the current in the excitation coil. The magnetic field, which is called the primary field, induces eddy current in the sample. Consecutively, a secondary magnetic field is induced by the eddy current and it opposes the primary field. This secondary field is then detected by a sensing device, which typically can be either a magnetic sensor or a coil. The output signal of the sensing device is then passed to the next stage to be conditioned and processed where eventually features are extracted in order to infer the desired parameters, such as wall thickness and lift-off, from the testing.

From one implementation to another, the systems vary primarily because of the differences in the excitation signal, excitation system, sensing device and the signal processing and feature extraction techniques. These variations are discussed below.

\subsection{Excitation Signals}

In many implementations, the excitation current or voltage is a square waveform. In some other applications, the excitation is of rectangular waveform which allows a very high power to be delivered in a limited duration in order not to overheat the coil and the driver electronics. And there are also other shapes of excitation signal that have also been used and proposed by researchers. A study on different excitation waveforms, namely square, half-sine and ramp, shows a favour for the square waveform [2]. A variable pulse width excitation has also been proposed [3], which was used in the inspection of subsurface corrosion in conductive structures [4]. Pulse width modulation, as illustrated in Fig. 5, provides different frequency spectra and is suggested of being able to eliminate the need for reference sample signal [5]. PEC has also been implemented by using the decaying part of the step signal, rather than the raising part, after the power supplied to the excitation coil is disconnected [6-8].

\subsection{Probes}

Typically, a PEC probe would contain one excitation coil and one or more sensing devices. An excitation coil generates primary transient excitation field, while one or more sensing devices picks up secondary eddy current field due to a sample. Probe designs are usually optimized in terms 


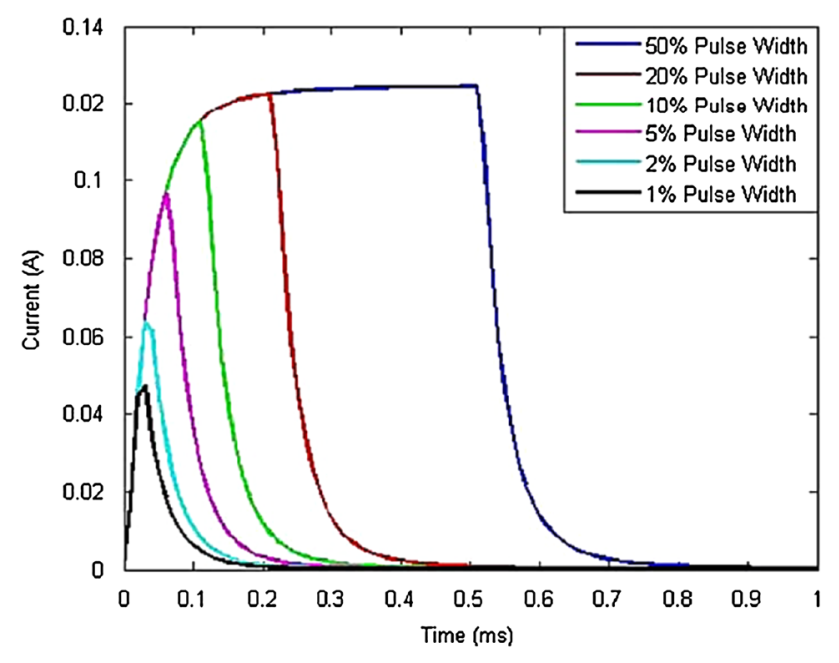

Fig. 5 Excitation currents with varied pulsed widths [5]

of its structure, the type of sensing elements and the use of cores based on the specific applications in which they will be deployed.

Based on the electromagnetic coupling between the excitation coil and the sample, eddy current probe's excitation coils can generally be categorized into one of the following three types: surface (or pancake) coil, encircling coil (or OD for outer diameter) and internal coil (also called bobbin or ID for inner diameter) [9]. The three types of coils are illustrated in Fig. 6. Surface or pancake coils may be orientated either parallel or normal to the surface of the sample and they are used for both flat and curved samples. Encircling coils are generally used in the inspection of cylindrical elongated structures, such as hollow pipes and solid rods [10], [11]. Coils of this type form a circle around the diameter of the test object coaxially. The specimen maybe insulated or coated. The bobbin-typed coils are usually used to inspect hollow cylindrical structures, such as pipes and bore holes, from the inside. In PEC NDT, a coil of this type which is used in remote field mode has been used for measurement of wall thickness of ferromagnetic tubes [12].

Differential probes, as opposed to absolute probes, are also used with the advantage of the self-nulling features and therefore no reference signals are required. This type of probe can be implemented by using two pick-up coils or two magnetic sensors with the output signal being the difference between the two output signals from the sensing devices. A differential double-D probe using two Hall devices has been investigated by Park, et al [13] which shows a potential for detection and sizing of sub-surface cracks in stainless-steel structures. Differential probes have also been studied for crack detection near a fastener in aircraft structures [14], [15].

Less common probe configurations have also been used, such as a planar matrix probe that can generate a color map that is useful in the identification of defects [16]. Their work shows the use of an 8-by-8 array of sensors, as shown in Fig. 7(a), successfully maps the surface defects that have been artificially made on the sample, which justifies the complexity of the excitation and sensing circuits used in the probe. The application of independent excitations lead to a more uniform excitation field which, in turn, leads to a simpler interpretation of the detected signals. Another interesting example of the use of sensor array in ECT, is shown in Fig. 7(b), where the printed array is flexible and can be used to produce a color map of surface corrosion [16]. Another unique example is a symmetric excitation coil introduced by Yang et al, which is expected to generate linear eddy currents with the benefit of virtually no field will be detected by the pick-up sensor when no defect is present [18].

Another differentiating feature is the shape of the coil. Rather than being circular, which is the most common shape, the coil may be rectangular or racetrack. This noncircular type of a coil is also referred as directional as opposed to non-directional or isotropic for circular coils. With directional probes, the paths of the induced eddy currents are not circular, and, therefore, they are more sensitive to changes in a particular direction. One example of the use of directional rectangular coil is the work done by He, et al [19], [20]. They also state that a more uniform eddy current distribution is being an advantage gained by using such a coil.

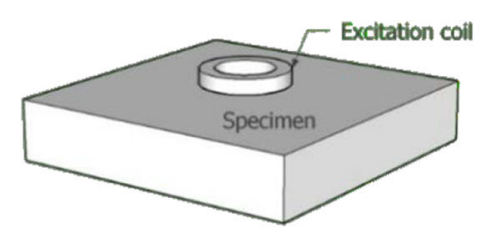

(a)

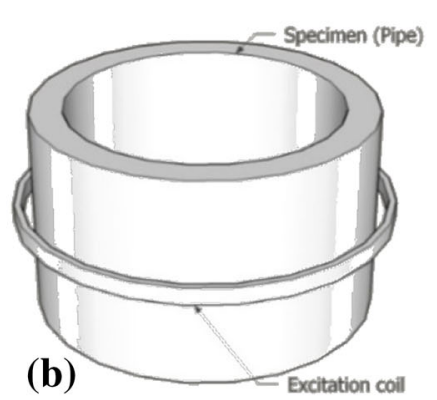

(b)

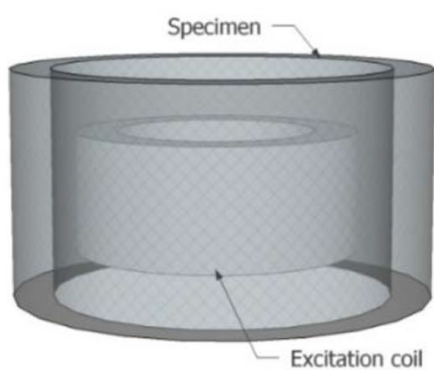

(c)

Fig. 6 Coil types used in ECT: (a) surface coil, (b) encircling coil, and (c) internal coil 


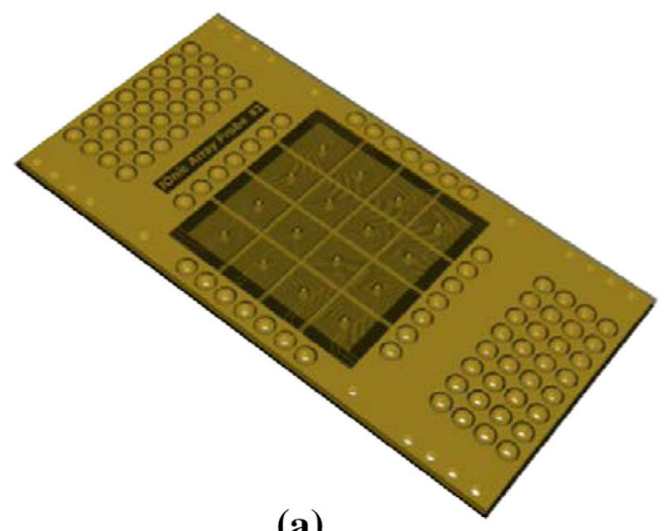

(a)

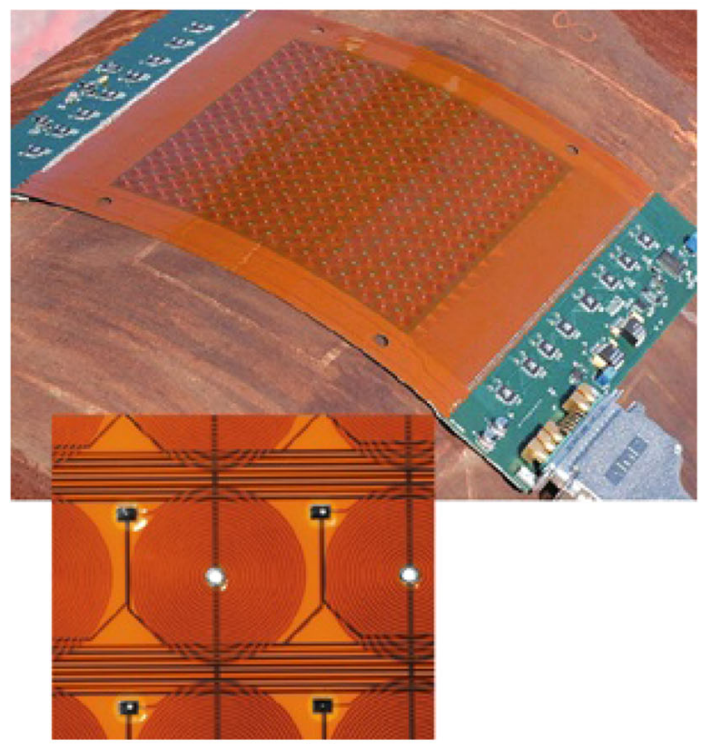

(b)

Fig. 7 Examples of arrayed sensors: (a) A planar matrix probe [16], (b) A flexible printed circuit array produced by Southwest Research Institute (adopted from [17])

A ferromagnetic core and shielding maybe present with the purpose of concentrating the magnetic flux and amplifying the signal. Hence, for enhancement of the sensitivity of the probe in certain applications, a ferromagnetic core or shielding is added to the probe. An example of the study in this topic is introduced in Ref. [21], where the sensitivity in sub-surface detection was evaluated. The study, which uses both numerical modelling and experimental tests, shows a favour for a ferrite-cored but unshielded over a shielded, ferrite-cored probe. A study by Zhou, et al [22] shows that a shield made of iron, that is positioned between the excitation coil and the magnetic sensor, provides a higher sensitivity compared with nonshielded and aluminium-shielded probes for ferromagnetic inspection.

\subsection{Sensing Devices}

While the excitation is always achieved by using an induction coil, the sensing devices can broadly be categorised into two different types, namely induction coils and magnetic sensors. The outputs of induction coils and magnetic sensors are given by

$V_{\text {coil }}=-N \cdot A \cdot \frac{\mathrm{d} B}{\mathrm{~d} t}$,

$V_{\text {mag_sen }}=K \cdot B$,

where $N$ is the number of the coil turns, $A$ is the area that magnetic flux passes through, $B$ is the magnetic field density, and $K$ is a coefficient of the magnetic sensor.

The output signal of an induction coil depends on the rate of change of magnetic flux density, while that of a magnetic sensor is directly proportional to magnetic flux density. As a result, the response from an induction coil exhibits similar characteristics with that of a magnetic sensor [23]. Each of these types has its own advantages and disadvantages. Induction coils play a dominant role in use for simplicity of operation and design, wide frequency bandwidth and large dynamics. However, induction coils are only sensitive to AC magnetic field, which is one of the drawbacks, although this drawback could be handled by introducing movement to the coil [24]. Magnetic sensors are typically sensitive to low frequency signals and offer a better spatial resolution. Hence, magnetic sensors have gained wide acceptance for enhancement of sensitivity and spatial resolution in sensing low frequency magnetic field [25]. In the applications where extremely high temperatures are involved, induction coils can be the only feasible option.

There are various types of magnetic field sensors available nowadays. Accumulatively, they can sense field from as low as in the order of several $10^{-15} \mathrm{~T}$ up to around 100 mT [26]. In PEC NDT, the most widely used magnetic sensors are Hall effect devices (e.g., Ref. [27]) and magnetoresistive (MR) devices. Both of these sensor types are relatively low cost. Hall effect devices have the highest dynamic range, covering approximately $100 \mu \mathrm{T}$ up to 100 $\mathrm{mT}$, however they have high noise, low resolution and low sensitivity.

Out of three existing types of MR technologies, two types are used in PEC NDT, which are anisotropic magnetoresistance or AMR(e.g., Ref. [28]) and giant magnetoresistance or GMR (e.g., Ref. [29]). The use of the latest development of MR, i.e., tunnelling magnetoresistance (TMR), in PEC systems has not been found by the authors in any literature despite it has the highest sensitivity. The lack of the presence if TMR in PEC systems may be due to its relatively new availability. While its technology is still 
being improved, an analysis - that may be rather outdated now - shows that despite its higher sensitivity compared with AMR and GMR, its detectivity is not necessarily higher due to its higher $1 / f$ noise [30]. The detectivity signifies the lowest magnetic field a sensor can detect, where the signal-to-noise ratio is unity. However, it is expected that further development of TMR will improve its detectivity and may make it more interesting for PEC applications.

Another type of magnetic field sensor, namely high temperature superconducting quantum interference device (HTS SQUID) magnetometer has also been studied in PEC [31]. SQUID has the highest sensitivity with the ability of measuring in the range down to $10^{-15} \mathrm{~T}$, however its applications in NDT is still limited due to its practicality and costs.

Whilst most of sensing devices used are absolute, the use of gradient-field GMR sensors has also been reported by $\mathrm{Li}$, et al [32]. Interestingly, they discover that the gradient-field measurements give better sensitivity and accuracy in detection and sizing artificial sub-surface corrosion in layered samples. Typically, two measurement points are deployed in gradient-field sensing. The two points stand at two different z-distances to the specimen under test.

Additionally, Joubert, et al have studied the use of magneto-optical (MO) film for imaging the structure of riveted multi-layered aircraft assembly for detecting cracks that are emanating from the rivet in hidden layers of the structure [33], [34]. The film is affected by the distribution of the magnetic fluxes induced by the eddy current and will reflect the light deliberately shone on it towards a CCD camera.

In terms of the direction of the field that is measured, most probes would detect field normal to the surface of the sample under test. However, there are also probes that are designed to detect the field parallel to the surface, such as the work in [35].

\section{Modelling}

PEC's electromagnetic problems, just like other eddy current NDT\&E methods, are governed by Maxwell equations. They can be solved in both time and frequency domains. For frequency domain solutions, the time-based signals are transformed first into frequency spectrum by using Fourier transform before later, having gone through some processing, transformed back into the time domain in order to get the final results. As with many other engineering problems, both analytical and numerical models are used in PEC. Analytical methods are known to be fast in the processing, although they are used only for relatively simple geometries. Reports show that researchers have attempted to build and use both analytical and numerical models for PEC since 1980's [36-39], with the initial objective of gaining better understanding of the PEC phenomena when used in NDT inspection. Therefore, the relative success in the modelling of PEC has allowed the advancement of PEC and its successful application to many different problems. In the following paragraphs, a few examples of works dealing with PEC modelling are mentioned with no intention for delving into details.

Recently, Desjardins, et al have presented an analytical approach [10], [40] to model an encircling coil used around a ferromagnetic rod. The method computes the time-domain electromagnetic response of the system and the results were validated by experimental data that display a very good agreement. Increasingly, a method for determining the electrical conductivity and the magnetic permeability that arises from the analytical solution for the ferromagnetic rod and encircling coils has been also suggested by Desjardins et al [11]. This is a good example of how modelling may enhance the use of PEC in NDT inspection.

An analytical method based on Truncated Region Eigenfunction Expansion (TREE) has been discussed in Ref. [27], which shows a good accuracy with experimental test data. The use of TREE method transforms the PEC model from an integral into a sum of series. Fig. 8 shows the flowchart of the proposed method. Moreover, this method extends the scope of analytical method significantly [41].

For the measurement of the wall thickness of pipes, another analytical method relying on the use of inverse Laplace transform has been developed in Ref. [42]. The model is, unlike most reported analytical models, non-axisymmetric and it presents a good correlation with measured data. Rather than having an encircling coil, the excitation coil is perpendicular to the surface of the pipe. For complex cases, numerical algorithms like Stehfest and Fast Fourier transform have been proposed to do the inversion of Laplace transform, because analytical inversion of Laplace transform is inaccessible [43]. Chen et al also proposed new signal features that can be used for determining the sample plate's magnetic permeability and electrical conductivity assuming that the thickness is previously determined by means of their analytical model [44].

An analytical model based on the Fourier superposition concept has been presented for modelling the crack detection task in a multi-layered structure and validated by using FEM results [45]. Accuracy of the model in the results has been shown with fast execution time compared to the FEM solution. An example of the use of numerical model is introduced in Ref. [46], which studies PEC signals when corrosion occurs in the inner wall of an insulated pipe. The signal features such as peak and zero crossing 


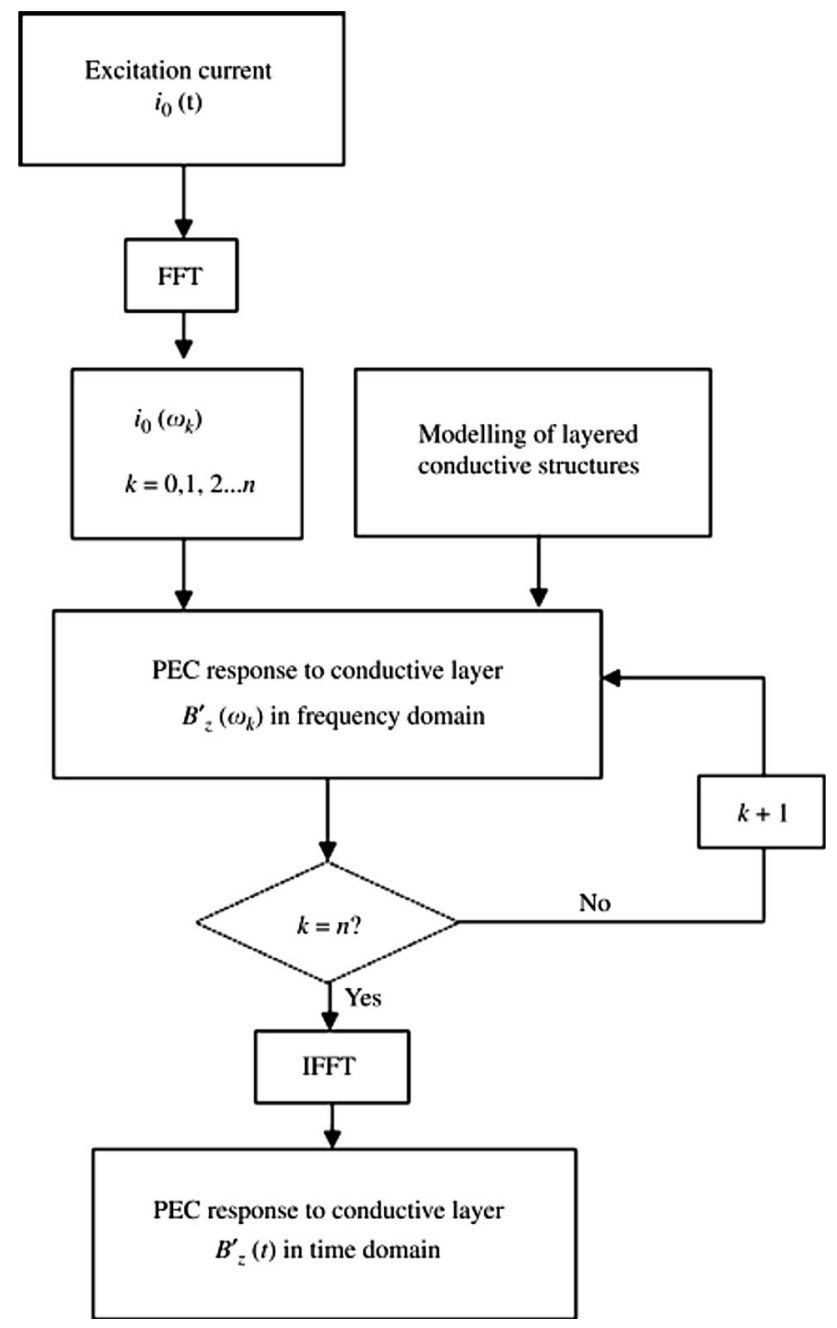

Fig. 8 A TREE-based modelling method for PEC [27]

times were investigated by using the FEM model. Another example of reported work on numerical was presented in Ref. [47] that used the Fourier transform rather than timestepping. The validation of the model was carried out by using experimental data at selected frequencies for detection of denting problems.

\section{Feature Extraction and Classification}

PEC signals, as conventional ECT, are affected by various factors, such as electrical conductivity, lift-off, magnetic permeability, thickness of the sample and inhomogeneity of the material. The other challenges faced by researchers in obtaining useful information from the signals are the noise and the low level signals in some cases. Consequently, the right signal processing, signal analysis, feature extraction and classification model must be implemented in order to attain the desired parameters, such as coating thickness, size and position of defects, and to isolate them from the undesired parameters, such as lift-off variation.

\subsection{Signal Feature and Feature Extraction}

In most of PEC techniques, a reference signal is used which is captured from a defect-free reference sample. Then a difference signal is obtained by subtracting the reference signal from the test one. Different types of signal are collected depending whether a coil or a magnetic sensor is used. A coil will capture the time-derivative of the magnetic field while a magnetic sensor will sense the field itself. PEC time responses can be normalized in order to reduce the effects of lift-off variation and varying magnetic permeabilities prior to the calculation of the above timedomain features [48]. Fig. 9 shows typical examples PEC signals obtained by using a Hall-device.

The features that are used to infer the desired parameters may be readily available, such as peak value [32], [48], [49], peak arrival time [49], rising time [50] and zero crossing time [51]. In addition to these more traditional features, there have also been emerging features proposed in various reports, such as the so called "relative variation of magnetic flux" [20], which has been shown through both simulation and experiments to have potentials in determining the lift-off for ferromagnetic materials [7]. This feature is calculated by processing an interval of the decay part of the field is used rather than the rising part and it should not, nevertheless, be used for small lift-offs. Huang et al discussed another feature derived from the cumulative integration of the time signal from the decaying part [8]. The feature is related to the time constant of the first order component of the signal and it is used to predict the

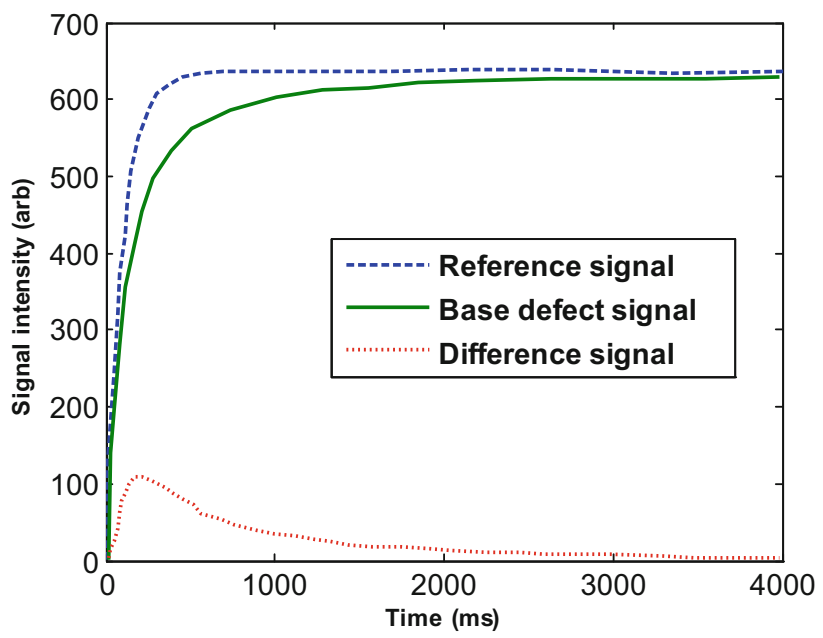

Fig. 9 Typical PEC signals obtained by using a Hall-device-based probe 
thickness of ferromagnetic plates. Another example is the kurtosis coefficient, which represents the craggedness level of a PEC response signal, that is used in sample's edge identification [52].

In other cases, researchers try to optimise the discrimination within the range of the parameters, which require them to employ data dimensionality reduction techniques. In some of the cases, the obtained features are subsequently fed into a classifier in order to either classify or quantify the defects.

Dimensionality reduction techniques used in the feature extraction for PEC signals that have been reported include principal component analysis (PCA), independent component analysis (ICA) [53] and Fisher linear discriminant analysis (FLDA) [51]. PCA has been the most widely used since the work of Tian, et al that was reported in [54]. Other examples of work where PCA has been used are introduced in Refs. [14], [28], [48], [52], [55-60].

The input data that are fed into the feature extraction stage can be time domain signals, frequency domain signals or the combination of both domains. Examples of time domain signal input are captured in Refs. [28], [54], [14].

In addition to the time domain features, frequency domain features are also utilized, both with and without the use of the time domain ones. This is understandable as PEC signals contain a wide spectrum of frequencies. And considering the skin depth effect, it is widely known that higher frequencies are more sensitive to surfer or near surface defects, while lower frequencies penetrated deeper resulting in sensitivity to more deeply buried defects.

For classification of surface and sub-surface defects, He, et al [19] used amplitudes of a few harmonics generated by using FFT. In another more recent work, for mapping artificial surface slots and holes, Abrantes, et al have also used FFT to get the amplitudes and phases of the fundamental and harmonics [16]. By using the amplitudes and phases of selected frequencies, the defects can be identified. The phases are shown to pinpoint the centre of the defect when they swing by $180^{\circ}$. The very high frequencies that are used should mean that only surface defects can be dealt with the approach.

Another example of FFT-based work is done in Ref. [56], which shows the ability to discriminate different types of defects, namely surface crack, sub-surface crack, surface cavity and sub-surface cavity. An improved result is, however, shown when the time signal is decomposed first by using wavelet into the approximate and detail signals before being transformed into the frequency domain by using the FFT. PCA is then used in order to extract the most significant features to be used for the classification stage.

Power spectral density analysis (PSDA) is also used by Qiu, et al [61] as frequency-domain features, which are believed by the authors to be more stable and accurate in the extraction than time domain features, citing the work [62] that uses spectral amplitude and phase as the signal features.

Finally, there are many works where both time and frequency domain features are used simultaneously. Within this group, time-frequency decomposition tools are often used, such as Wavelet, empirical mode decomposition (EMD) and Rihaczek distribution. Following the analysis, a feature extraction technique, such as PCA and Fisher linear discriminant analysis (FLDA), is then normally used in order to reduce the data dimensions for defect classification purposes.

Tian, et al used wavelet transform, which captures both temporal and spectral information from the time signals of PEC, in order to improve their classification [63]. Hosseini and Lakis use Rihaczek distribution for time-frequency analysis of the signals and follow it up using PCA to reduce the features, which are the used classify defects located in different layers in a multi-layered specimen [60].

The use of both time and frequency domains is studied by Ref. [18] for detection and characterization of cracks under fasteners in aircraft layered-structures. The combination of the signal analysis and unique symmetric excitation coil leads to the ability in detection of $1 \mathrm{~mm}$-long crack at the depth of $10 \mathrm{~mm}$ aided by a GMR sensor. Although in the study, the time and frequency domain features were presented separately, the combination of both may increase the confidence in the detection.

Zhang, et al combines both time domain features, namely peak value, peak time and rising time, together with a frequency domain feature derived by using EMD and Hilbert transform for classification of surface notch, sub-surface notches and wall losses [64]. In this work, the researchers do not need to use any data dimensionality reduction.

\subsection{Classifiers}

As mentioned earlier, in many cases researchers have proposed the use of a classifier for classification or quantification of defects. It is thought that this would be an important and desirable feature for future PEC systems that will potentially reduce the level of operator skill requirements. An automated system that will reliably provide information on the location and type or size of a detected defect would be advantageous which reduce dependency on human interpretation skills.

Broadly, there are two major groups of classification methods, which are supervised and unsupervised. In supervised classification, the training data are labelled based on the class, such as defect type or location. On the other hand, unsupervised classification does not require the labelling of the training data, therefore this classification is 
really only clustering the input data without identifying what each cluster represents.

Both categories of classification have been studied and used in PEC NDT. The supervised classification methods include support vector machine (SVM) and Linear Discriminant Analysis (LDA), whilst the used unsupervised include K-means and Bayes. In the majority of the reported works, neither classifying nor clustering methods have been used. Rather, manual human interpretation is made on the plots of distribution of the features.

There are only a few reports on the use of supervised classification, one of them is Ref. [51], where SVM techniques have been employed in conjunction with Fischer's LDA (FLDA) for localization and classification of defects in multi-layered structure.

For unsupervised methods, K-means has been used for estimation of the size the cracks, also in aircraft structures [28]. Another report by Qiu, et al [61] included both supervised and unsupervised methods, namely LDA and Bayes. Although they report both approaches managed to separate the surface and sub-surface defects, from the resulting plots it can be seen that the separation achieved by using LDA is better and has relatively wider distances between the two groups of defects.

\subsection{Lift-off Problem}

Lift-off problem has always been associated with eddy current NDT, and PEC is not an exception. The detrimental effect of lift-off slows down and limits the spread of the applications of PEC and ECT in NDT. Thankfully, many researchers have attempted to overcome this problem and proposed techniques to eliminate or reduce the effect of lift-off variation. Each of these techniques may only be applicable to specific PEC system implementations and applications.

The most popular features to be used in order to minimize the effects of lift-off is called lift-off point of intersection (LOI), which is a point at which PEC signals intersect when only lift-off distance varies. Therefore, LOI is inherently immune to lift-off variations, which makes a LOI point one of desirable features to remove lift-off effect for PEC evaluation. LOI phenomenon was originally observed in experiments and proposed to avoid false indication due to lift-off effect when material loss in a twolayer assembly was evaluated by PEC technique [65], [66]. It is reported that the signals of LOI points have been successfully adopted to measure the thickness of a plate [67] and quantify defects and their locations in a layered sample [68]. To enable the interpretation of LOI signals, the characteristics of LOI signals in terms of time and amplitude were revealed under a wide variety of conditions. The results demonstrate that LOI signals including both the time and amplitude follow a unique curve against thickness and conductivity [69]. At the early stage, LOI phenomenon could only exist when a PEC probe using a coil as the receiver is placed above a nonferrous sample. Tian, et al [70] obtained LOI points by doing the derivative of the output of a Hall-based probe, and $\mathrm{Li}$ [71] extended the method to a PEC probe in which a solid-state magnetic field sensor could be placed at arbitrary positions. Further, Lefebvre and Mandache [72] recorded LOI phenomenon by covering a conductive but nonferrous layer on a ferromagnetic substrate. Also, Mandache and Lefebvre [73] made an attempt to disclose the physics of the LOI phenomenon. It is found that the LOI phenomenon is a general characteristic of the eddy currents, not only to transient eddy currents. Kral, et al [74] presented a linear transformer model to investigate the origin of LOI phenomenon, and obtained LOI points from the time derivatives of the output of a GMR-based probe. Interestingly, a LOI point is recently observed in the spectral PEC signals [67].

The use of normalisation and of two reference signals has been proposed to reduce the effects of lift-off [75]. Tian, et al found that lift-off invariance (LOI) existed for magnetic sensors - not only for pick-up coils - and proposed new lift-off estimation techniques by using theoretical models [70]. They believe that the techniques may also be used when an array of pick-up sensors are used.

A technique to indirectly reduce the effects of lift-off by using the slope of the difference signal has been proposed [76]. The technique is potentially useful in the measurement of depth of surface defect.

The work done by Huang and Wu shows that the relative rate of change of the magnetic flux is independent of liftoff and, therefore, is a good candidate for getting the desired information from the PEC signals, which was in the inference of the thickness of ferromagnetic plates [77]. The use of normalisation in frequency domain signals can also be used to reduce the effect of lift-off variation [78].

\section{NDT\&E Applications of PEC}

Thanks to its versatility, PEC NDT has been used in numerous different NDT applications, both in material characterization and structural integrity inspection. In material characterization, PEC has been suggested to be used for measurement of electrical conductivity and magnetic permeability of materials. Whilst, in the structural integrity testing, PEC NDT has been applied for defect detection and characterization, evaluation of corrosion, measurement of insulation thickness, plate thickness and wall thickness of pipe. This covers both insulated and noninsulated, coated and non-coated materials. Furthermore, still within the area of integrity testing, detection of cracks 
under fasteners and between fasteners in aircraft structures using PEC has also been explored and implemented. References to show the wide diversity of PEC applications are included and discussed briefly below.

\subsection{Measurement of Electrical Conductivity and Magnetic Permeability}

The use of PEC in the measurement of electrical conductivity of ferromagnetic materials, such as carbon steel plates, has been investigated by Chen, et al [44]. They have successfully established PEC as a non-contacting measurement method with a maximum error of $1.6 \%$. In a similar application, Desjardins, et al [10] mentioned in their study that the resulting conductivity from the transient eddy current is within $0.5 \mathrm{MS} / \mathrm{m}$ from that obtained by the four-point measurement. A carbon steel tube with wall thickness of 0.035 " was used in their experiment, which was also supported by an analytical calculation with an excellent agreement. In the same work, they also showed a good result on the use of PEC in measurement of magnetic permeability.

\subsection{Measurement of Thickness of Insulation and Coating}

In ensuring its efficiency, the thickness of thermal insulation of vessels and pipes is an important parameter that needs to be monitored regularly in petrochemical industries. Experimental measurements of the thickness of thermal insulation on a steel plate has been done by using PEC and the report shows that an effective thickness measurement of up to $80 \mathrm{~mm}$ is possible [7].

In the area of coating thickness determination, Tai, et al obtained excellent results for both magnetic and nonmagnetic coatings on both magnetic and non-magnetic substrates by using peak values [79]. Their work was also supported by analytical theoretical models. Coating is very important for corrosion protection, among others.

\subsection{Measurement of Thickness and Evaluation of Corrosion}

Thickness measurement and evaluation of corrosion are discussed in the same section as basically the work on the same principle and the many of the works done in thickness measurement are intended for inspection of corrosion.

An example of the work in plate thickness measurement is introduced in Ref. [8], where a thickness of up to $30 \mathrm{~mm}$ was shown to be successfully measured. The plate was made of Q235 steel. Another example of work in this topic was presented by Shin, et al who investigated different materials, including copper, tungsten, inconel 600, aluminium and titanium [80].

Röntgen Technische Dienst(RTD) - now known as Applus RTD - has created a PEC system called RTDIncotest that is able to measure the wall thickness - ranging from $6 \mathrm{~mm}$ up to $65 \mathrm{~mm}$ - of both pipes and plates made of low alloy carbon steel, even when they are under insulation of up to $150 \mathrm{~mm}$ in thickness [81]. By measuring the wall thickness, the corrosion under insulation (CUI) can be evaluated.

Another company - Canada-based Eddyfi - has also made a commercial PEC system more recently. The system is capable of measuring wall thickness of up to $64 \mathrm{~mm}$ under non-magnetic and non-conductive insulation with thickness up to $203 \mathrm{~mm}$ [82]. The testing can even be performed in the presence of conductive weather jackets of up to $1.5 \mathrm{~mm}$ thick (depending on the material). The ability of measuring wall thickness through the insulation and weather jacket is clearly a unique advantage for PEC systems.

Research works related to the estimation of pipe wall thickness include [42], [49], [83], and [84]. A potentially effective method based on time-to-peak has been presented by $\mathrm{Xu}$, et al for the assessment of wall thinning of insulated pipe [49]. The time-to-peak feature divided by the thickness of a calibration sample is linearly related to wall thickness within $60 \%$ of the calibration sample [49]. Such a feature is robust to thermal insulation and lift-off, which is verified by Park, et al [85]. In a very similar type of application, Park et al studied the use of PEC to determine the wall thinning of stainless steel pipes in nuclear power plants without removing the insulation, where it could estimate thickness of up to $5 \mathrm{~mm}$ at the lift off of $6 \mathrm{~mm}$ [84]. Huang, et al [8] and Cheng investigated the decay behaviour of logarithmic scaled PEC signals. It is found that the decay coefficient is approximately linear to wall thickness, and is insensitive to lift-off variations [29].

A distinctive approach by using a remote field probe has been presented in Ref. [12] for wall thickness measurement of ferromagnetic tubes from the inside. The researchers believe that this would be suitable for harsh-environment operation.

\subsection{Detection and Characterization of Defects}

There is a long list of literatures covering this topic, showing that it has been the main focus of researchers in PEC. For example, detection and sizing of sub-surface defects in a non-magnetic specimen made of AISI type 316 stainless steel has been shown in Ref. [21]. The deepest notch was located $6 \mathrm{~mm}$ below the surface in an $8-\mathrm{mm}$ thick block. Works with similar types of artificial defects 
and similar shape of sample, i.e., plate or block, have also been reported by Tian, et al [50], [54], [63], [86].

Other examples of defect detection and characterization include flaws in the inner surface of pipe under insulation [87], outer corrosion on carbon steel pipes used in oil and gas industry [35], surface and sub-surface cracks and cavity detection in con-casting slabs (CCS) [56], subsurface corrosion in a plate [4], surface corrosion on coated mild steel S275 plates [48], [88], and various types of defect on steam generator support structures [59]. Detection of wall thinning in pipe structures has been included in the previous sub-section above.

Aircraft industry has been given a lot of attention by the PEC research community since the very early stages of PEC history. Stress-induced cracks that occur under a fastener and between fasteners in multi-layered structures of the wings are crucial to be detected early for the safety of the aircraft during its flights. Works related to these issues can be found in the following works about PEC [1], [14], [15], [18], [28], [58], [60], [89], among others.

Related to the aircraft industry is the new emerging material carbon fiber reinforced plastic or CFRP for short. CFRP is growing in use in various industrial sectors thanks to its strength and lightness. However, its relative proneness to impact damage immediately leads to the industrial requirement of effective NDT techniques for ensuring its integrity. On the light of this, researchers have investigated the application of eddy current NDT, including PEC, to detect and characterize defects and damage in CFRP [90], [91]. The CFRP problem is particularly challenging due to its anisotropic characteristic. It can be said that the achievement in this application is still limited.

An initial study of the possibility of measuring stress in aluminium alloy has been reported in Ref. [92], which concludes that further work should be done before the technique can be realized as an inspection tool. Another application in defect detection is one for friction stir welding [93].

\section{Challenges and Opportunities}

The potential opportunities of PEC are shaped by both its strengths and the progressively more quantitative requirements of NDT\&E, which is characterised by ever increasingly more stringent safety regulations; and this is especially true in the sectors where human lives are in stake, such as aerospace and nuclear industry. This would make the role of NDT\&E even more important and relevant than ever. Opportunities for NDT in general and PEC in particular, are also presenting themselves as currently many structures and aircraft fleet are ageing, which need suitable and appropriate NDT inspections for ensuring safe and reliable operations with minimum downtimes.

A reliable, yet affordable technique will obviously be preferred by customers and operators alike. It is thought that the costs of the application of NDT can be reduced through enhanced sensitivity and improved user-friendliness, among others. With improved sensitivities, the interval between tests can be made longer and, therefore less tests need to be performed, which in turn, will help reduce the costs. This challenge presents an opportunity for PEC, especially in the optimisation and innovation related to the probe design, which should make the best use both analytical and numerical modelling methods. An example is the use a rather innovative a differential probe based on a rectangular excitation coil [94], that has been designed with the help of a finite element model. The probe successfully produces higher sensitivity in detection of defect in different materials, including CFRP.

The sensitivity of the PEC techniques may also be improved through the use of newer types of magnetic sensors, such as the tunnelling magnetoresistance (TMR), thanks to the continuous improvement in the sensitivity of magnetic sensing technologies. TMR exhibits a higher sensitivity and higher signal-to-noise ratio (SNR) compared with hall devices and other magnetoresisitive sensors, including GMR [95].

As for more user-friendly systems, they would be depending less on the high skills of the operators for interpreting the signals and the amount of training can be kept a minimum. All this, again, will help save the costs involved in in-service inspection for ensuring the integrity of structures or parts. Such systems can be materialized by having an automated classification for defects. The use of supervised classification techniques should be studied and developed more in order to give an answer to this challenge. Applications that may benefit from an automated classification functionality are those where multi types or locations of defects are potentially present, which include the inspection of aircraft multilayer structures and inspection of support structures in steam generators.

Cost reduction can also be achieved when the inspection time can be lowered. In this case, PEC may be able to benefit from the usage of multi-modal NDT and data fusion. This approach has not shown any significant appearance in the literature and new research can be undertaken to open up new opportunities for the application of PEC NDT. Another way of slashing the inspection time is, as already rising in popularity with some other NDT techniques, by the application of robotics. Again, opportunities in this area of research are still wide open for exploration, while there is only a handful of relevant existing literature. 
In manufacturing industry, there is an increasing support for the use of NDT\&E to improve the quality and consistency of the products. The potential ability of contactless thickness measurement of plates or sheets should be exploited so that PEC with its unique benefits can be used in manufacturing process control for such products. The ability of negating the effects of lift-off variations during measurement has to be achieved for generating consistently accurate results. If the required level of accuracy can be met, the PEC approach can be more advantageous compared with, for example, the use of laser triangulation sensors that would need access to both sides of the plates or sheets.

Simultaneous measurement of multiple parameters, such as conductivity, permeability and thickness is strongly demanding, and attracting increasingly more attention for quality control or structural integrity in some cases. In practice, multi-sensing modalities are performed for a comprehensive assessment, which is costly, labour-intensive and sometimes time-consuming. Fortunately, PEC is thought to be a feasible and efficient tool to measure conductivity, permeability and thickness simultaneously. Chen, et al [44] proposed an analytical-model-based method to infer conductivity and permeability with the prerequisite of knowing the wall thickness beforehand. Adewale, et al [96] investigated the contribution of permeability and conductivity on PEC signals. It is found that conductivity effects are prominent in the rising edge of the transient response, whereas permeability effects dominate in the steady stage. This finding presents a feasible way to decouple permeability and conductivity, and implies a feasible method to infer these two parameters within one measurement. Desjardins, et al [10] created a novel forward-solutions-based inverse method for simultaneous and accurate characterization of permeability and conductivity by computing the areas under scaled PEC signals. At present, the developed methods are only proven to be feasible. However, much work still needs to be done to make these methods applicable. For example, the developed methods need to be extended in order to be able to determine four or more parameters or be applied to a layered sample. Further, the accuracy of the existing methods greatly relies on the correlation between calculated and experimental data. The efforts to reduce the discrepancy should be made for accuracy improvement. In addition, uncertainty analysis should also be performed to evaluate the proposed methods [97].

\section{Conclusions}

PEC NDT\&E has received a great deal of attention from researchers in the past 30 years and more, and yet, new applications and techniques are still being proposed and reported, as shown in this review. This gives approval to the theoretical understanding that PEC is rich of information that is potentially useful for quantitative NDT\&E.

Literature on different aspects of PEC NDT has been presented and discussed. Challenges and opportunities of PEC NDT in the future have also been brought forward. It is expected that this work can contribute to research work in PEC NDT, especially for researchers and developers who are embarking a project in PEC NDT.

Acknowledgements The authors would like to thank Ministry of Higher Education of Malaysia for funding the project on PEC NDT at IIUM through the research grant FRGS16-059-0558. This work is also supported by the National Natural Science Foundation of China under research grants 51677187 and 51307172. Dr. Mengbao Fan would like to thank China Jiangsu Provincial Department of Education and China University of Mining and Technology for sponsoring his visit to Newcastle University as an academic visitor.

\section{References}

1. Lebrun B, Jayet Y, Baboux J-C. Pulsed eddy current signal analysis: application to the experimental detection and characterization of deep flaws in highly conductive materials. NDT E Int., 1997, 30(3): 163-170.

2. Michniakova M, Janousek L. The impact of various waveform shapes on the response signal in pulsed eddy current NDE. ELEKTRO 2012, 2012: 439-442.

3. Li J, Wu X, Zhang Q, et al. Pulsed eddy current testing of ferromagnetic specimen based on variable pulse width excitation. NDT E Int., 2015, 69: 28-34.

4. Li Y, Yan B, Li D, et al. Pulse-modulation eddy current inspection of subsurface corrosion in conductive structures. NDT E Int., 2016, 79: 142-149.

5. Abidin I Z, Mandache C, Tian G Y, et al. Pulsed eddy current testing with variable duty cycle on rivet joints. NDT E Int., 2009, 42(7): 599-605.

6. Dolabdjian C P, Perez L, De Haan V O, et al. Performance of magnetic pulsed-eddy-current system using high dynamic and high linearity improved giant magnetoresistance magnetometer. IEEE Sensors Journal, 2006, 6(6): 1511-1517.

7. Li J, Wu X, Zhang Q, et al. Measurement of lift-off using the relative variation of magnetic flux in pulsed eddy current testing. NDT E Int., 2015, 75: 57-64.

8. Huang $\mathrm{C}$, Wu X. An improved ferromagnetic material pulsed eddy current testing signal processing method based on numerical cumulative integration. NDT E Int., 2015, 69: 35-39.

9. Blitz J. Eddy current testing of metails. NDT E Int., 1997, 30(1): 41.

10. Desjardins D, Krause T W, Clapham L. Transient eddy current method for the characterization of magnetic permeability and conductivity. NDT E Int., 2016, 80: 65-70.

11. Desjardins D R, Krause T W, Clapham L. Transient response of a driver coil in transient eddy current testing. NDT E Int., 2015, 73: $8-14$

12. Vasic D, Bilas V, Ambrus D. Pulsed eddy-current nondestructive testing of ferromagnetic tubes. IEEE Transactions on Instrumentation and Measurement, 2004, 53(4): 1289-1294.

13. Park D G, Angani C S, Cheong Y M. Differential pulsed eddy current probe to detect the sub surface cracks in a stainless steel pipe. 18th World Conference on Nondestructive Testing, 2012. 
14. Horan P, Underhill P R, Krause T W. Pulsed eddy current detection of cracks in F/A-18 inner wing spar without wing skin removal using Modified Principal Component Analysis. NDT E Int., 2013, 55: 21-27.

15. He Y, Luo F, Pan M, et al. Pulsed eddy current technique for defect detection in aircraft riveted structures. NDT E Int., 2010, 43(2): 176-181.

16. Abrantes R F, Rosado L S, Piedade M, et al. Pulsed eddy currents testing using a planar matrix probe. Measurement, 2016, 77: 351-361.

17. Southwest Research Institute. Southwest Research Institute 2004 Annual Report, 2004.

18. Yang G, Tamburrino A, Udpa L, et al. Pulsed eddy-current based giant magnetoresistive system for the Inspection of aircraft structures. IEEE Transactions on Magnetics, 2010, 46(3): 910-917.

19. He Y, Pan M, Luo F, et al. Pulsed eddy current imaging and frequency spectrum analysis for hidden defect nondestructive testing and evaluation. NDT E Int., 2011, 44(4): 344-352.

20. He Y, Luo F, Pan M, et al. Defect classification based on rectangular pulsed eddy current sensor in different directions. Sensors Actuators A Phys., 2010, 157(1): 26-31.

21. Arjun V, Sasi B, Rao B P C, et al. Optimisation of pulsed eddy current probe for detection of sub-surface defects in stainless steel plates. Sensors Actuators A Phys., 2015, 226: 69-75.

22. Zhou D, Wang J, He Y, et al. Influence of metallic shields on pulsed eddy current sensor for ferromagnetic materials defect detection. Sensors Actuators A Phys., 2016, 248: 162-172.

23. Fan M, Cao B, Sunny A I, et al. Pulsed eddy current thickness measurement using phase features immune to liftoff effect. NDT E Int., 2017, 86: 123-131.

24. Tumanski S. Induction coil sensors-a review. Meas. Sci. Technol., 2007, 18(3): R31-R46.

25. Ripka $\mathrm{P}$, Janosek M. Advances in magnetic field sensors. IEEE Sensors Journal, 2010, 10(6): 1108-1116.

26. Herrera-May A L, Aguilera-Cortés L A, García-Ramírez P J, et al. Resonant magnetic field sensors based on MEMS technology. Sensors, 2009, 9(10): 7785.

27. Li Y, Tian G Y, Simm A. Fast analytical modelling for pulsed eddy current evaluation. NDT E Int., 2008, 41(6): 477-483.

28. Kim J, Yang G, Udpa L, et al. Classification of pulsed eddy current GMR data on aircraft structures," NDT E Int., 2010, 43(2): 141-144.

29. Cheng W. Pulsed eddy current testing of carbon steel pipes' wallthinning through insulation and cladding. J. Nondestruct. Eval., 2012, 31(3): 215-224.

30. Albrecht J, Carls S, Robert S. Magnetoresistive Sensors for Nondestructive Evaluation. 10th SPIE International Symposium, Nondestructive Evaluation for Health Monitoring and Diagnostics, Conference 5770, 2005.

31. Panaitov G, Krause H-J, Zhang Y. Pulsed eddy current transient technique with HTS SQUID magnetometer for non-destructive evaluation. Phys. C Supercond., 2002, 372-376: 278-281.

32. Li Y, Yan B, Li D, et al. Gradient-field pulsed eddy current probes for imaging of hidden corrosion in conductive structures. Sensors Actuators A Phys., 2016, 238: 251-265.

33. Joubert P Y, Le Diraison Y, Xi Z, et al. Pulsed eddy current imaging device for non destructive evaluation applications. Sensors, 2013 IEEE, 2013: 1-4.

34. Joubert P Y, Le Diraison Y. Pulsed eddy current imager for the enhanced non destructive evaluation of aeronautical riveted assemblies. IEEE SENSORS 2014 Proceedings, 2014: 1188-1191.

35. Majidnia S, Nilavalan R, Rudlin J. Investigation of an encircling Pulsed Eddy Current probe for corrosion detection. IEEE Sensors 2014 Proceedings, 2014: 835-838.
36. Dai X W, Ludwig R, Palanisamy R. Numerical simulation of pulsed eddy-current nondestructive testing phenomena. IEEE Transactions on Magnetics, 1990: 26(6): 3089-3096.

37. Ludwig R, Dai X W. Numerical and analytical modeling of pulsed eddy currents in a conducting half-space. IEEE Transactions on Magnetics, 1990: 26(1): 299-307.

38. Allen B L, Lord W. Finite element modeling of pulsed eddy current phenomena. Review of Progress in Quantitative Nondestructive Evaluation, 1984: 3(A): 561-568.

39. Allen B, Ida N, Lord W. Finite element modeling of pulse Eddy current NDT phenomena. IEEE Transactions on Magnetics, 1985, 21(6): 2250-2253.

40. Desjardins D P R, Krause T W, Gauthier N. Analytical modeling of the transient response of a coil encircling a ferromagnetic conducting rod in pulsed eddy current testing. NDT E Int., 2013, 60: 127-131.

41. Fan M, Huang P, Ye B, et al. Analytical modeling for transient probe response in pulsed eddy current testing. NDT E Int., 2009, 42(5): 376-383.

42. Chen X, Lei Y. Time-domain analytical solutions to pulsed eddy current field excited by a probe coil outside a conducting ferromagnetic pipe. NDT E Int., 2014, 68: 22-27.

43. Fan M, Cao B, Wang Y. Computation of coil-induced voltage due to a defect-free plate using Stehfest's method for pulsed eddy current evaluation. Insight Non-Destructive Test. Cond. Monit., 2010, 52(6): 302-304.

44. Chen X, Lei Y. Electrical conductivity measurement of ferromagnetic metallic materials using pulsed eddy current method. NDT E Int., 2015, 75: 33-38.

45. Theodoulidis T, Wang H, Tian G Y. Extension of a model for eddy current inspection of cracks to pulsed excitations. NDT E Int., 2012, 47: 144-149.

46. Liu S X, Xin W, Ding K Q. Simulation of corrosion on detection for pulsed eddy current. Fuzzy Systems and Knowledge Discovery (FSKD), 2010 Seventh International Conference on, 2010, 4: 1839-1842.

47. Pavo J. Numerical calculation method for pulsed eddy-current testing. IEEE Transactions on Magnetics, 2002, 38(2): 1169-1172.

48. Alamin M, Tian G Y, Andrews A, et al. Principal component analysis of pulsed eddy current response from corrosion in mild steel. IEEE Sensors Journal, 2012, 12(8): 2548-2553.

49. $\mathrm{Xu} \mathrm{Z}, \mathrm{Wu} \mathrm{X}, \mathrm{Li} \mathrm{J}$, et al. Assessment of wall thinning in insulated ferromagnetic pipes using the time-to-peak of differential pulsed eddy-current testing signals. NDT E Int., 2012, 51: 24-29.

50. Tian G Y, Sophian A. Defect classification using a new feature for pulsed eddy current sensors. NDT \& E International, 2005, 38(1): 77-82.

51. Chen X, Hou D, Zhao L, et al. Study on defect classification in multi-layer structures based on Fisher linear discriminate analysis by using pulsed eddy current technique. NDT E Int., 2014, 67: $46-54$.

52. BinFeng Y, FeiLu L, Dan H. Research on edge identification of a defect using pulsed eddy current based on principal component analysis. NDT E Int., 2007, 40(4): 294-299.

53. Yang G, Tian G Y, Que P W, et al. Independent component analysis-based feature extraction technique for defect classification applied for pulsed eddy current NDE. Res. Nondestruct. Eval., 2009, 20(4): 230-245.

54. Sophian A, Tian G Y, Taylor D, et al. A feature extraction technique based on principal component analysis for pulsed Eddy current NDT. NDT \& E International, 2003, 36(1): 37-41.

55. Horan P F, Underhill P R, Krause T W. Real time pulsed eddy current detection of cracks in F/A-18 inner wing spar using discriminant separation of modified principal components analysis scores. IEEE Sensors Journal, 2014, 14(1): 171-177. 
56. Qiu X, Zhang P, Wei J, et al. Defect classification by pulsed eddy current technique in con-casting slabs based on spectrum analysis and wavelet decomposition. Sensors Actuators A Phys., 2013, 203: 272-281.

57. Pan M, He Y, Tian G, et al. Defect characterisation using pulsed eddy current thermography under transmission mode and NDT applications. NDT E Int., 2012, 52: 28-36.

58. Stott C A, Underhill P R, Babbar V K, et al. Pulsed eddy current detection of cracks in multilayer aluminum lap loints. IEEE Sensors Journal, 2015, 15(2): 956-962.

59. Buck J A, Underhill P R, Mokros S G, et al. Pulsed eddy current inspection of support structures in steam generators. IEEE Sensors Journal, 2015, 15(8): 4305-4312.

60. Hosseini S, Lakis A A. Application of time-frequency analysis for automatic hidden corrosion detection in a multilayer aluminum structure using pulsed eddy current. NDT E Int., 2012, 47: 70-79.

61. Qiu X B, Liu L L, Li C L, et al. Defect classification by pulsed eddy-current technique based on power spectral density analysis combined with wavelet transform. IEEE Trans. Magn., 2014, 50(9): $1-8$.

62. Kiwa T, Kawata T, Yamada H, et al. Fourier-transformed eddy current technique to visualize cross-sections of conductive materials. NDT E Int., 2007, 40(5): 363-367.

63. Tian G Y, Sophian A, Taylor D, et al. Wavelet-based PCA defect classification and quantification for pulsed eddy current NDT. IEE Proceedings - Science, Measurement and Technology, 2005, 152(4): 141-148.

64. Zhang Q, Chen T, Yang G, et al. Time and frequency domain feature fusion for defect classification based on pulsed eddy current NDT. Res. Nondestruct. Eval., 2012, 23(3): 171-182.

65. Giguère S, Lepine B A, Dubois J M S. Pulsed eddy current technology: characterizing material loss with gap and lift-off variations. Res. Nondestruct. Eval., 2001, 13(3): 119-129.

66. Giguère S, Dubois S J M. Pulsed eddy current: Finding corrosion independently of transducer lift-off. AIP Conf. Proc., 2000, 509(1): 449-456.

67. Angani C S, Ramos H G, Ribeiro A L, et al. Lift-off point of intersection feature in transient eddy-current oscillations method to detect thickness variation in stainless steel. IEEE Transactions on Magnetics, 2016, 52(6): 1-8.

68. Liu Z, Ramuhalli P, Safizadeh S, et al. Combining multiple nondestructive inspection images with a generalized additive model. Meas. Sci. Technol., 2008, 19(8): 817-822.

69. Lefebvre J H V, Dubois J M S. Lift-off point of intercept (LOI) behavior. AIP Conference Proceedings, 2005, 760: 523-530.

70. Tian G Y, Li Y, Mandache C. Study of lift-off invariance for pulsed eddy-current signals. IEEE Trans. Magn., 2009, 45(1): 184-191.

71. Li Y B, Chen Z B, Qi Y. Generalized analytical expressions of liftoff intersection in PEC and a liftoff-intersection-based fast inverse model. IEEE Trans. Magn., 2011, 47(10): 2931-2934.

72. Lefebvre J H V, Mandache C. Pulsed eddy current thickness measurement of conductive layers over ferromagnetic substrates. Int. J. Appl. Electromagn. Mech., 2008, 27(1-2): 1-8.

73. Mandache $\mathrm{C}$, Lefebvre J H V. Transient and harmonic eddy currents: Lift-off point of intersection. NDT E Int., 2006, 39(1): 57-60.

74. Kral J, Smid R, Ramos H M G, et al. The lift-off effect in Eddy currents on thickness modeling and measurement. IEEE Trans. Instrum. Meas., 2013, 62(7): 2043-2049.

75. Tian G Y, Sophian A. Reduction of lift-off effects for pulsed eddy current NDT. NDT E Int., 2005, 38(4): 319-324.

76. Yu Y, Yan Y, Wang F, et al. An approach to reduce lift-off noise in pulsed eddy current nondestructive technology. NDT E Int., 2014, 63: 1-6.
77. Huang $\mathrm{C}, \mathrm{Wu} \mathrm{X}$. Probe lift-off compensation method for pulsed eddy current thickness measurement. Antennas and Propagation (APCAP), 2014 3rd Asia-Pacific Conference on., 2014: 937-939.

78. Tian G Y, He Y, Adewale I, et al. Research on spectral response of pulsed eddy current and NDE applications. Sensors Actuators A Phys., 2013, 189: 313-320.

79. Yang H-C, Tai C-C. Pulsed eddy-current measurement of a conducting coating on a magnetic metal plate. Meas. Sci. Technol., 2002, 13(8): 1259.

80. Shin Y-K, Choi D-M, Kim Y-J, et al. Signal characteristics of differential-pulsed eddy current sensors in the evaluation of plate thickness. NDT E Int., 2009, 42(3): 215-221.

81. Robers M A, Scottini R. Pulsed eddy currents in corrosion detection. NDT.net, 2002: 7(10).

82. Eddyfi. Lyft Specifications Sheet. [Online]. Available: http:// www.eddyfi.com/wp-content/uploads/2016/09/specificationssheet-lyft.pdf. [Accessed: 02-Oct-2016].

83. Xie S, Chen Z, Takagi T, et al. Quantitative non-destructive evaluation of wall thinning defect in double-layer pipe of nuclear power plants using pulsed ECT method. NDT E Int., 2015, 75: 87-95.

84. Park D G, Angani C S, Kim G D, et al. Evaluation of pulsed eddy current response and detection of the thickness variation in the stainless steel. IEEE Transactions on Magnetics, 2009, 45(10): 3893-3896.

85. Park D-G, Angani C S, Kishore M B, et al. The effects of lift-off from wall thinning signal in pulsed eddy current testing. $J$. Magn., 2012, 17(4): 298-301.

86. Chen T, Tian G Y, Sophian A, et al. Feature extraction and selection for defect classification of pulsed eddy current NDT. NDT E Int., 2008, 41(6): 467-476.

87. Lai S, Chen D Y, Chen H, et al. Pulsed eddy current testing of inner wall flaws in pipe under insulation. Procedia Eng., 130: $1658-1664$

88. Tian G, He Y, Alamin M, et al. Corrosion characterisation using pulsed eddy current sensor systems. Sensing Technology (ICST), 2011 Fifth International Conference on., 2011: 498-503.

89. Sophian A, Tian G Y, Taylor D, et al. Design of a pulsed eddy current sensor for detection of defects in aircraft lap-joints. Sensors Actuators, A Phys., 2002: 101(1-2): 92-98.

90. Wu J, Zhou D, Wang J, et al. Surface crack detection for carbon fiber reinforced plastic (CFRP) materials using pulsed eddy current testing. Nondestructive Evaluation/Testing (FENDT), 2014 IEEE Far East Forum on., 2014: 181-185.

91. He Y, Tian G, Pan M, et al. Non-destructive testing of low-energy impact in CFRP laminates and interior defects in honeycomb sandwich using scanning pulsed eddy current. Compos. Part B Eng., 59: 196-203.

92. Morozov M, Tian G Yun, Withers P J. The pulsed eddy current response to applied loading of various aluminium alloys. NDT E Int., 2010, 43(6): 493-500.

93. Rosado L S, Santos T G, Ramos P M, et al. A differential planar eddy currents probe: Fundamentals, modeling and experimental evaluation. NDT E Int., 2012: 51: 85-93.

94. Zhou D, Wang $\mathrm{J}$, Wu J, et al. Investigation of rectangular differential probes for pulsed eddy current non-destructive testing. Insight - Non-Destructive Test. Cond. Monit., 2016, 58(2): 87-100.

95. Sergeeva-Chollet N, Decitre J, Fermon C, et al. Intrinsic noise study of Eddy current probes based on magnetoresistive sensors. 11th European Conference on Non-Destructive Testing (ECNDT 2014), 2014.

96. Adewale I D, Tian G Y. Decoupling the influence of permeability and conductivity in pulsed eddy-current measurements. IEEE Transactions on Magnetics, 2013, 49(3): 1119-1127. 
97. Fan M, Cao B, Yang P, et al. Elimination of liftoff effect using a model-based method for eddy current characterization of a plate. NDT E Int., 2015, 74: 66-71.

Ali Sophian, born in 1974 in Indonesia, is currently an Assistant Professor with Mechatronics Engineering Department at the Faculty of Engineering, International Islamic University Malaysia, Gombak, Malaysia. He received his B.Eng and $\mathrm{PhD}$ in Electronics Engineering from the University of Huddersfield, UK in 1998 and 2003 respectively. He has published more than 20 papers, mainly in electromagnetic NDT as the outcomes of the research projects funded by the TWI Ltd, EPSRC (UK) and Universities UK. Currently, he is leading research projects in non-destructive testing (NDT), Sensors and Image Processing funded mainly by Malaysia's Ministry of Higher Education (MOHE).

Guiyun Tian, born in 1965, received his B.Sc. degree and M.Sc. degreefrom University of Sichuan, Chengdu, China in 1985 and 1988, respectively, and Ph.D. from University of Derby, Derby, UK, in 1998. He is currently the Professor of Sensor Technologies at School of Electrical and Electronic Engineering, Newcastle University, UK. He is also adjunct professor in the School of Automation Engineering, University of Electronics Science and Technology of China. His research interests include electromagnetic sensors, sensor array and sensor network, electromagnetic non-destructive evaluation, advanced signal processing and integrative systems and applications.
He has coordinated several research projects from the Engineering and Physical Sciences research Council (EPSRC), Royal Academy of Engineering and FP7. Also he has good collaboration with leading industrial companies, such as Airbus, Rolls Royce, BP, nPower, Network Railand TWI among others. He has published more than 300 papers in Journals and peer-reviewed conferences with h-index 42.

Mengbao Fan, was born in Shandong China, in 1981. He received his B.Sc. degree in automation from the China University of Petroleum, China, and Ph.D degree in control science and engineering from Zhejiang University, China, in 2004 and 2009, respectively. Since 2009 to 2011, he was a Lecturer with the School of Mechatronic Engineering, China University of Mining and Technology. Since 2012, He has been an associate professor with the School of Mechatronic Engineering, China University of Mining and Technology. From January 2015 to January 2016, he academically visited the School of Electrical and Electronic Engineering, Newcastle University, Newcastle upon Tyne, UK. His current research interests include nondestructive testing and evaluation for quality control and structural integrity, modeling of eddy current field and computation, sensors and instrumentation. He is an author of more than 40 technical papers on journals and conference proceedings. He has coordinated multiple research projects, such as National Natural Science Foundation of China, and Jiangsu Provincial Natural Science Foundation of China, and also has good collaboration with industries. 